\title{
Multiproxy approach to reconstruct the Pliocene climate
}

\author{
Antoni Rosell-Melé, E.L. McClymont ${ }^{2}$, P.S. Dekens ${ }^{3}$, H. Dowsett ${ }^{4}$, A.M. Haywood ${ }^{5}$ and C. Pelejero ${ }^{6}$
}

Barcelona, Catalonia, Spain, 17-19 September 2014

The Pliocene epoch has often been proposed as a climate analogue for future conditions on Earth. However, despite relatively small differences in climate control factors, including atmospheric $\mathrm{CO}_{2}$ concentration, the Pliocene climate was markedly different from the modern climate. This has made the Pliocene a relevant target for validating climate models. This in turn requires confidence in the paleoclimate estimates to be able to fully ascertain model strengths and weaknesses. In this context, and in order to further develop efforts by earlier projects such as PRISM (Pliocene Research, Interpretation and Synoptic Mapping group) and PlioMIP (Pliocene Model Intercomparison Project), about 65 specialists of the international community of Pliocene researchers met at a workshop in Barcelona. The key mission was to establish guidelines to facilitate further a community wide international effort to reconstruct key climatic parameters (temperature, $\mathrm{CO}_{2}$, continental and sea ice, sea level, vegetation) in selected time intervals within the Pliocene epoch. The ultimate goal of this community effort is to provide a comprehensive global representation of Pliocene climate, which would facilitate data modeling comparisons. To this end, the workshop consisted of invited plenary talks that synthesized the current state of the art, followed by working group discussions of research priorities, and reporting and synthesis presentations towards the end of the workshop. Participants presented their current research in poster sessions.

Plenary talks highlighted the challenges of trying to reconstruct Pliocene climate and the need to reassess some concepts. These included the recognition that the Pliocene does not fit the paradigm of a "stable climate", nor should it be considered an isolated time period, but instead part of a climate continuum, preceded by the much warmer Miocene. Thus, no specific time slice within the Pliocene will be representative of the epoch's entire range of climate variability, while the character of Pliocene interglacials could be as variable as those of the Quaternary. Integrating or comparing models and data requires dealing with reconstructions with very different constraints on the time (e.g. series or slices with no time lapse) and spatial domains (e.g. global vs regional), all with their own uncertainties. Among the many implicit challenges, urgency was placed on the need to revise the existing Pliocene marine isotope reference templates on which time series age models are based, and the quantification of uncertainty in proxy reconstructions.

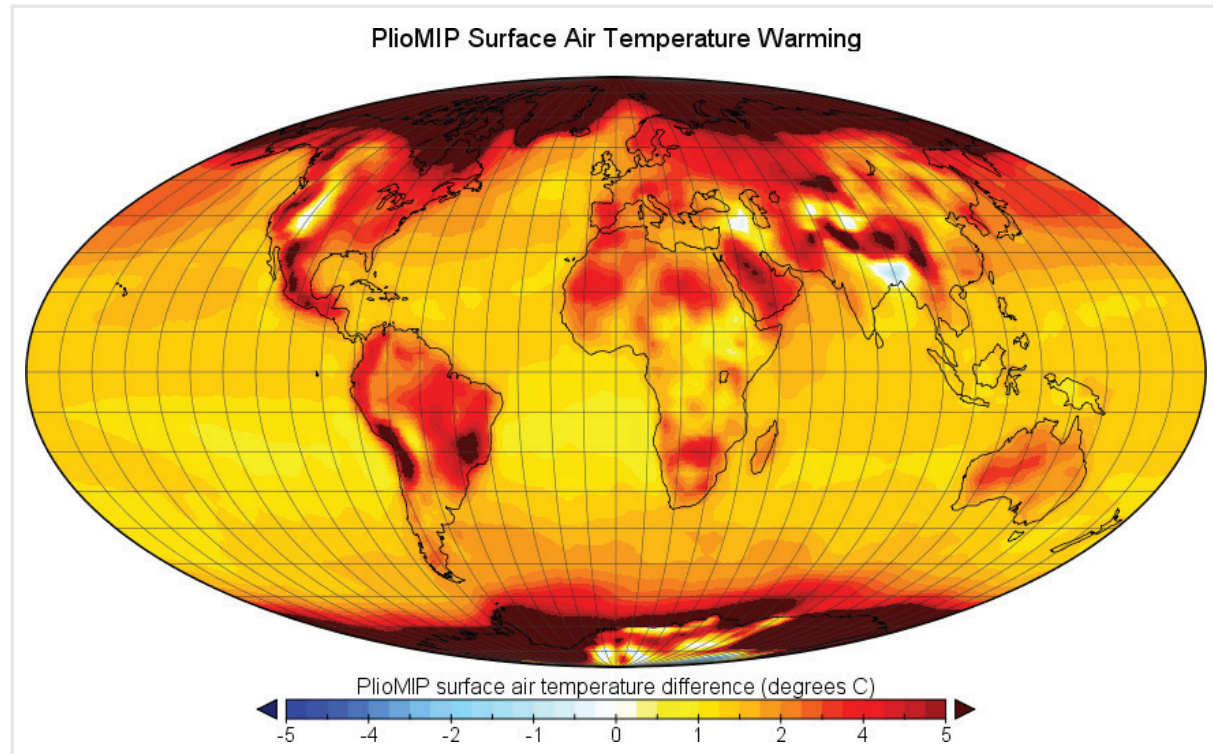

Figure 1: Mean annual surface air temperature anomaly (mid-Pliocene minus pre-industrial simulations) based on a multi-model means ensemble obtained by the Pliocene Model Intercomparison Project. Adapted from Haywood et al. (2013).

From the discussions, a consensus emerged on setting research priorities for different time lines. For instance, in the short-term, there is a need to focus efforts on the reconstruction of isotopic stages M2 through to $\mathrm{KM} 3$ (i.e. ca. 3.2-3.3 Ma) and on the improvement of the spatial and temporal reconstructions of proxy data sets to constrain meridional temperature gradients and conditions in high latitude environments.

In the mid-term, one of the priorities is to further the development and application of proxies and models, for instance related to precipitation, or the disentanglement of multiple environmental effects affecting our proxies of temperature and sea-level. It is also paramount to target key regions and issues on which information is lacking, such as the reconstruction of sea-ice or continental precipitation.

In the long term, efforts should focus on creating syntheses that include both relative changes (e.g. information on forcings) and absolute changes required for quantitative data-model integration, for time periods that include the early Pliocene and eventually provide space and time transect information. The priorities will be available in a more elaborate form on the workshop web site: http://jornades.uab.cat/plioclim/.

The next step of the initiative is to seek its consolidation through the creation of a formal working group, and summarize some of the discussion in research papers. We also aim to create a database to summarize the proxy data and make them accessible for further research such as data-model comparisons. The group agreed to review and reassess its objectives in a meeting in two years, to be held in Norway.

\section{ACKNOWLEDGEMENTS}

The organizers thank PAGES, ICREA, EGU, and SCARPAIS for their financial support, ICTA-UAB for administrative support, and ICM-CSIC for allowing the use of their facilities for the workshop.

\section{AFFILIATIONS}

'ICREA and Institute of Environmental Science and Technology, Universitat Autònoma de Barcelona, Catalonia, Spain

${ }^{2}$ Department of Geography, Durham University, UK ${ }^{3}$ Department of Earth \& Climate Sciences, San Francisco State University, USA

${ }^{4}$ Eastern Geology \& Paleoclimate Science Center, US Geological Service, Reston, USA

${ }^{5}$ School of Earth and Environment, University of Leeds, UK

${ }^{6}$ CREA and Institut de Ciències del Mar, CSIC, Barcelona, Catalonia, Spain

\section{CONTACT}

Antoni Rosell-Melé: antoni.rosell@uab.cat

\section{REFERENCE}

Haywood AM et al. (2013) Clim Past 9: 191-209 